\title{
锂离子电池石墨负极微结构数值重建及特征化分析
}

\author{
何绍阳 ${ }^{1,2}$, 曾建邦 ${ }^{1}$, 蒋方明 ${ }^{1}$ \\ (1. 中国科学院 广州能源研究所先进能源系统实验室, 广州 510640; 2. 中国科学院大学, 北京 100049)
}

摘 要: 实际锂离子电池石墨负极由鳞片状石墨层叠而成, 具有明显的各向异性。本文基于椭球颗粒的模拟退火法 对其进行三维微结构数值重建。重建微结构乃三相(孔或电解液、石墨和固体添加物)复合结构, 很好地再现了实际 电极各向异性特征。对重构电极进行特征化分析，得到了电极内固/孔相的连通率、比表面积以及孔径分布等信息; 发现石墨颗粒尺寸对电极特性有重要的影响：椭球形石墨颗粒尺寸越大，则重建微结构的平均孔径越大，比表面 积越小; 相对于赤道半径，椭球颗粒的极半径对重建电极特性的影响更为明显。

关＼cjkstart键＼cjkstart词：锂离子电池；石墨负极；微结构重建；模拟退火法；椭球颗粒

中图分类号: TQ174 文献标识码: A

\section{Numerical Reconstruction and Characterization Analysis of Microstructure of Lithium-ion Battery Graphite Anode}

\author{
HE Shao-Yang ${ }^{1,2}$, ZENG Jian-Bang ${ }^{1}$, JIANG Fang-Ming ${ }^{1}$
}

(1. Laboratory of Advanced Energy Systems, Guangzhou Institute of Energy Conversion, Chinese Academy of Sciences, Guangzhou 510640, China; 2. University of Chinese Academy of Sciences, Beijing 100049, China)

\begin{abstract}
The real graphite anode of lithium-ion battery is of evident non-isotropic characteristic due to its cascading graphite flakes. An ellipsoid particle-based simulated annealing method is developed to numerically reconstruct the three-dimensional microstructure of graphite anode. The reconstructed anode is a composite of three clearly distinguished phases: pore (or electrolyte), graphite and solid additive, well representing the non-isotropic characteristic of real graphite anode. Characterization analysis of reconstructed electrodes gives information such as the connectivity, the specific surface area of solid or pore phase, and the pore size distribution. The results show that the size of graphite ellipsoids has important effects on the characteristics of electrode: 1) larger size graphite ellipsoids result in larger mean pore size and smaller specific surface area in the reconstructed electrode; 2) changing the polar radius of graphite ellipsoid particles has more pronounced influence on the characteristics of electrode than has changing its equatorial radius.
\end{abstract}

Key words: lithium-ion battery; graphite anode; microstructure reconstruction; simulated annealing method; ellipsoid particles

锂离子电池与传统的二次电池如镍镉、铅酸电 池等相比, 具有电压高、比能量大及循环寿命长等
优点, 因此, 它在数码、移动通讯以及新能源汽车等 行业得以广泛应用。然而, 目前单体锂离子电池性

收稿日期：2015-01-26; 收到修改稿日期：2015-04-15

基金项目：国家自然科学基金(51206171); 广州市科技计划项目(y409zc1001); 中国科学院 “百人计划” 项目 National Natural Science Foundation of China (51206171); Guangzhou Scientific and Technological Program (y409zc1001); Chinese Academy of Sciences One Hundred Talent Program

作者简介：何绍阳(1990-)，男，硕士研究生. E-mail: hesy@ms.giec.ac.cn

通讯作者: 蒋方明, 研究员. E-mail: jiangfangming@ms.giec.ac.cn 
能还不够理想, 导致动力或储能电池组的性能还不 能完全满足大规模应用要求, 安全性亦有待改善 ${ }^{[1]}$ 。 例如, 过充时, 负极极有可能因锂沉积反应的发生 生成锂枝晶, 造成电池内部短路, 从而导致安全事 故 ${ }^{[2-3]}$ 。有研究表明, 除了电极材料本身的因素外, 锂离子电池电极的复杂微孔结构对电池性能也具有 重要影响 ${ }^{[4-5]}$ 。

通过数值建模对锂离子电池内的物理-化学过 程进行描述和分析, 已经在锂离子电池的相关应用 研究和优化设计中发挥作用。大多数模型着眼于宏 观过程, 对电池细微尺度传输机理的研究和电池内 部介观微结构的设计优化难有作为。介观尺度模型 基于电极的微孔结构, 模拟和预测介观尺度传输现 象 $^{[6-8]}$ 。在锂离子电池介观尺度模型中, 各位置的材 料明确区分, 物性可根据相应的材料准确地设计; 固相活性物和电解液的交界面明确界定, 电化学反 应及锂原子或离子的嵌入和脱出过程得以更为准确 地描述和处理, 因此可以更好地研究和分析电极 (包括隔膜)介观微结构对电池内耦合电化学反应的 多物理传输的影响。介观尺度模型可以在现有材料 的基础上, 对电池电极(包括微孔构形)进行设计和 优化, 提升电池性能。并且基于重建微结构, 通过介 观尺度模型, 可以预测电极的有效传输(包括电极 内离子/电子的传输)物性, 分析获得材料颗粒的大 小、形态和体积分数等参数对电极性能的影响, 对 于改进 $\mathrm{LiFePO}_{4}$ 电极(材料本征导电性差)的性能亦 可能具有重要参考意义。

微结构重建是介观尺度模型的基础和前提。现 有数值重建方法主要有高斯随机场 ${ }^{[9]}$ 、模拟退火 (Simulated Annealing, 简称 SA) ${ }^{[10-14]}$ 、过程重建 ${ }^{[15]}$ 及随机网格 ${ }^{[16-17]}$ 等方法。其中, SA 法以能反应组分 空间分布的两点相关函数来构建目标函数, 该函数 可视为系统“能量”, 通过“能量”局部最小化 (即退火) 演化, 寻得系统能量的最小状态, 进而得到与实际 电极结构最接近的重建微结构状态。相比其它方法, SA 法不仅可以考虑更多的微结构统计信息, 而且 还可方便地处理固体活性物与添加物之间的空间分 布和粘接关系。Yeong 和 Torquato ${ }^{[10]}$ 首先提出了基 于体元的 SA 法, 算法执行的基本单元是重构区域 内的离散体元; Čapek 等 ${ }^{[11]}$ 从多孔介质中抽取两点 相关函数作为描述微结构特征的统计信息并以此作 为重建的重要输入参数; 在 Yeong 和 Torquato 提出 的方法基础上, Kim 等 ${ }^{[12]}$ 开发了基于均匀圆球颗粒 的 SA 法, 从而重建了质子交换膜燃料电池的催化 层的微结构; 吴伟等在均匀圆球颗粒模型的基础上,
进一步开发了颗粒直径满足一定分布函数的 SA 法, 分别对锂离子电池正极 ${ }^{[13]}$ 和质子交换膜燃料电池 的催化层 ${ }^{[14]}$ 进行了数值重建。

在吴伟等 ${ }^{[13]}$ 开发的、用于重建锂离子电池正极 的 SA 方法基础上，并考虑负极石墨颗粒的鳞片状 形态, 本工作开发出基于椭球颗粒的 SA 法, 数值重 构了石墨电极的微结构形貌, 并进行特征化分析, 深入探讨椭球颗粒尺寸对电极微结构特性的影响。

\section{1 石墨电极的特征和数值描述}

吴伟等 ${ }^{[13]}$ 在用 SA 方法重建锂离子电池正极时 假设有: 电极微结构各向同性和固体活性物呈球 状颗粒。这些假设对石墨负极可能不适用, 这主要 是因为与正极典型的颗粒堆积状微结构不同 (见图 1(a)), 负极石墨颗粒呈明显的鳞片状 (见图 1(b)), 通过导电剂、增稠剂和粘结剂等相互粘连在一起, 具有较明显的各向异性, 这从文献[18]中可以得到 验证。

在重建微结构中, 将导电剂、增稠剂和粘结剂 等全部视为固体添加物, 整个电极则简化为孔隙、 石墨和固体添加物组成的三相复合结构。电极重构 区域划分为一系列网格体元, 利用相函数进行描述:

$$
I_{i}(r)=\left\{\begin{array}{cc}
1 & r \in \text { phase } i \\
0 & \text { otherwise }
\end{array}\right.
$$

其中, $\boldsymbol{r}(x, y, z)$ 是三维空间(石墨电极重构区域)中任 意位置矢量, $i=0,1,2$ 。其中 0 表示孔相, 1 代表石墨 颗粒, 2 是固体添加物相。

通过统计平均可以得到各相的相应信息: 孔隙 率 $\varepsilon_{0}=<I_{0}(r)>$, 各组元体积分数 $\varepsilon_{i}=<I_{i}(r)>$, 两点相关 函数 $f_{i j}\left(r_{1}, r_{2}\right)=<I_{i}\left(r_{i}\right) I_{j}\left(r_{j}\right)>$ 。两点相关函数 $f_{i j}\left(r_{1}, r_{2}\right)$ 是在重构区域内的 $r_{1} 、 r_{2}$ 位置分别是 $i$ 相和 $j$ 相的概 率, 代表微结构中各相之间的空间分布关系。当假 定电极各向同性时, 两点相关函数简化为两点间距 离的函数, 但在本研究中, 以沿石墨电极厚度的方

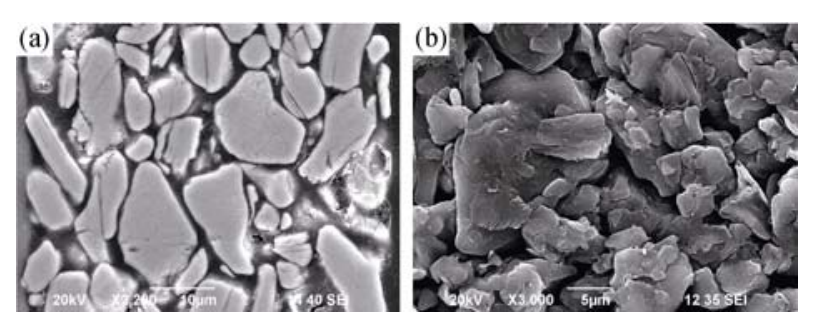

图 1 锂离子电池电极的 FIB/SEM 照片

Fig. 1 FIB/SEM images of lithium-ion battery electrodes (a) $\mathrm{LiCoO}_{2}$ cathode; (b) Graphite anode 
向为 $X$ 方向, 其它分别为 $Y 、 Z$ 方向。对于石墨电极 的微结构形态, 在 $X$ 方向具有明显的各向异性, 而 $Y 、 Z$ 两个方向可近似为各向同性, 因此本研究需要 设置 $\overline{r_{1} r_{2}}$ 与 $X$ 方向垂直的两点相关函数 $f_{i j}^{1}\left(r_{1}, r_{2}\right)$ 和 与 $X$ 方向平行的两点相关函数 $f_{i j}^{2}\left(r_{1}, r_{2}\right)$, 将它们转 换为两点距离 $u$ 的函数:

$$
\begin{cases}f_{i j}^{1}\left(r_{1}, r_{2}\right)=f_{i j}^{1}(u), & x_{1}=x_{2} \\ f_{i j}^{2}\left(r_{1}, r_{2}\right)=f_{i j}^{2}(u), & y_{1}=y_{2} \text { 且 } z_{1}=z_{2}\end{cases}
$$

文中基于椭球颗粒重构电极微结构, 共需要 6 个参数来描述椭球颗粒的形态, 其中以极半径 $a$ 和 两个赤道半径 $b$ 和 $c$ (其中 $c \geqslant b$ ) 来描述椭球的尺寸, 外加椭球 3 个轴的旋转方向角 $\alpha 、 \beta$ 和 $\gamma$ 。石墨颗粒 的两个赤道半径约为 $2.5 \sim 12.5 \mu \mathrm{m}$, 长短赤道半径之 比为 1.4 4.0, 极半径约为 1 3 $\mu \mathrm{m}^{[19-20]}$ 。在制备石墨 负极的工艺中, 颗粒沿电极厚度方向堆叠, 形成层 片状的结构特征。因此, 在数值重建过程中须对粗 球各个轴的旋转方向角加以限制, 将椭球极半径方 向设为电极厚度方向(即 $X$ 轴方向), 以 $X$ 轴为中心 的旋转方向角 $\alpha$ 的范围为 $\left(0,360^{\circ}\right)$; 以另外两轴为 中心的旋转方向角 $\beta 、 \gamma$ 在 $\left(-20^{\circ}, 20^{\circ}\right)$ 的范围内, 如 图 2 所示。

在重建过程中, 以实际电极的孔隙率、组元体积 分数、颗粒尺寸分布、两点相关函数等特性参数作为 输入参数; 以 $1 \mu \mathrm{m}$ 作为基本节点的尺寸, 重构的空间 区域节点数位 $100 \times 100 \times 100$ (即 $100 \mu \mathrm{m} \times 100 \mu \mathrm{m} \times$ $100 \mu \mathrm{m})$; 以彭鹏等 ${ }^{[21]}$ 的研究工作中同型号的锂离 子电池石墨负极为研究对象, 孔隙率为 0.301 、石墨 体积分数 0.523 , 添加剂体积分数 0.176 。对如图 1(a) 所示的石墨负极 FIB/SEM 切片图进行图像处理获 取两点相关函数时, 由于在切片图中难于区分出石
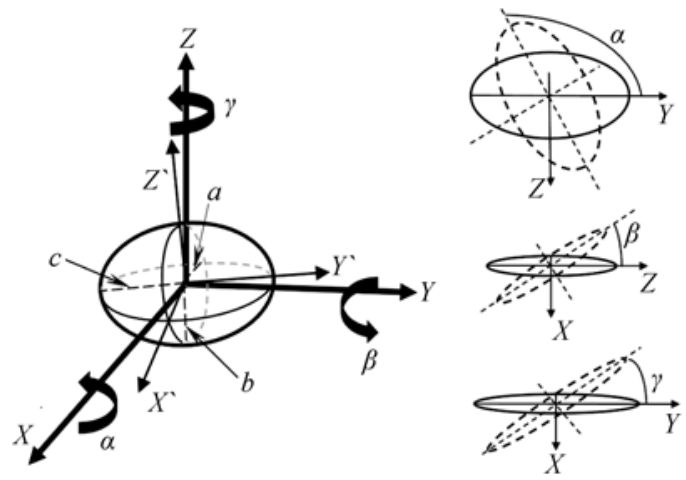

图 2 椭球颗粒

Fig. 2 Ellipsoid particle

The $a, b$ and $c$ are the three semi axial lengths of ellipsoid. The $\alpha, \beta$ and $\gamma$ are the three rotation angles for the $X^{\prime}-Y^{\prime}-Z^{\prime}$ coordinates relative to the $X-Y-Z$ coordinates
墨与固体添加物, 但固体添加物基本都是粘接在石 墨表面，因此假定图 1(a)中的所有固体均为石墨 颗粒，故可提取孔隙和石墨两相的两点相关函 数。在此基础上, 本课题组还假定半径为 $1 \mu \mathrm{m}$ 的 圆球形固体添加剂颗粒随机均匀地分布在石墨 颗粒表面上。

\section{2 基于椭球颗粒的 SA 法重建步骤}

基于椭球颗粒的 SA 法重建石墨负极微结构, 首先重构出石墨颗粒的空间分布, 然后再添加固体 添加物，具体重建步骤如下:

1) 椭球颗粒(石墨颗粒)系统初始化。将预设尺 寸大小的椭球颗粒随机放置在重建区域内, 并按一 定概率接收，接收概率按下式计算:

$$
P(v)=\exp \left(-\frac{v}{\varepsilon_{v}}\right)
$$

其中 $v$ 是当前放置颗粒与已有颗粒重叠部分体积的 占比, $\varepsilon_{v}$ 是调节重叠程度的常数。该步骤直到重建 区域内石墨相体积分数达到预设值时结束, 初始 化完成。

2) 随机移动椭球颗粒。随机选择一个椭球颗粒, 按随机产生的距离 $\mathrm{d} \boldsymbol{r}(\mathrm{d} x, \mathrm{~d} y, \mathrm{~d} z)$ 移动该颗粒, $\mathrm{d} x 、 \mathrm{~d} y$ 和 $\mathrm{d} z$ 均满足相同的指数分布, 以 $\mathrm{d} x$ 为例:

$$
P(\mathrm{~d} x)=\left\{\begin{array}{cc}
\frac{1}{a_{x} \varepsilon_{x}} \exp \left(-\frac{\mathrm{d} x}{\varepsilon_{x}}\right) & \mathrm{d} x \leqslant L_{x} \\
0 & \mathrm{~d} x>L_{x}
\end{array}\right.
$$

式中 $a_{x}=1-\exp \left(-L_{x} / \varepsilon_{x}\right), \varepsilon_{x}$ 是控制移动距离的常数, $L_{x}$ 是重建区域在 $X$ 方向的长度。

3) 判断是否接收步骤 2) 的移动。接受概率 $P$ 为:

$$
P(\Delta E)=\left\{\begin{array}{cc}
\exp \left(-\frac{\Delta E}{T}\right) & \Delta E \geqslant 0 \\
1 & \Delta E<0
\end{array}\right.
$$

式中 $T$ 为系统“退火温度”; $\Delta E$ 为移动前后系统能量 值的变化量, 用于作为系统能量的目标函数的定 义为:

$$
E=\sum_{i=1}^{n} \sum_{u=0}^{U}\left(f_{i}(u)-f_{i}^{0}(u)\right)^{2}
$$

式中 $n$ 是重建区域体元总数, $U$ 为两点 $r_{1} 、 r_{2}$ 距离 $u$ 的最大值, $f_{i}(u) 、 f_{i}^{0}(u)$ 分别是重建电极过程中三 维微结构的两点相关函数和从二维 FIB/SEM 图片 提取的作为参照的两点相关函数, 其定义见式(2)。 由于石墨电极的各向异性,故我们以 $X$ 轴方向的 $f_{i j}^{1}\left(r_{1}, r_{2}\right)$ 、和 $Y, Z$ 轴方向的 $f_{i j}^{2}\left(r_{1}, r_{2}\right)$ 分别构建两个 能量值 $E_{1} 、 E_{2}$, 并计算移动前后系统两能量值的变 
化量 $\Delta E_{1} 、 \Delta E_{2}$, 用于计算概率 $P_{1} 、 P_{2}$, 取两概率值 中较小的一个判断是否接收该次移动。

4) 重复步骤 2)、3), 重复过程“退火温度” $T$ 以 冷却率 $\lambda=0.99$ 由初始值 $T_{0}$ 降到 $T_{\text {end }}$, 当 $T$ 达到目 标温度 $T_{\mathrm{end}}$, 终止计算过程, 得到石墨相三维微结 构骨架。

5) 将定直径的固体添加物圆球颗粒随机均匀 布置在石墨颗粒表面上, 直到重建区域内固体添加 物相体积分数达到预设值时结束。

\section{3 结果分析}

\section{1 重建结果}

基于椭球颗粒的 SA 法对锂离子电池石墨负极 进行微结构重建, 椭球颗粒的极半径和长、短赤道 半径分别设定为 $2 / 8 / 12 \mu \mathrm{m}$ 。重建过程先对石墨相执 行 SA 算法, 获得石墨电极的非均匀层片状微结构, 见图 3(a)。然后将固体添加物添加到重构区域中, 获 得其在重构区域的分布图, 见图 3(b)。最后得到界 限清晰且包含孔隙、石墨以及固体添加物的电极三 组元复合微结构, 见图 3(c)。

重建结构中，石墨相与实际电极切片 FIB/SEM 图像极为相似, 见图 1(a), 均呈现由鳞 片状的石墨颗粒沿电极厚度方向彼此堆叠的各向 异性结构。由于在重建过程中假定固体添加物颗 粒与石墨相有重叠时, 才接受该颗粒的添加, 从 而使得添加物颗粒紧贴在石墨表面, 与石墨有较 好的接触。该重建结构, 在一定程度上可以较好 地表现负极层状石墨颗粒与固体添加物经混合、 压实后所形成的骨架，与基于圆球颗粒的 SA 法 ${ }^{[11]}$ 相比, 基于椭球颗粒的 SA 法重建的石墨负极微 结构与实际更为接近。

\section{2 特征化分析}

特征化分析主要包括分析电极内固相或孔相连 通性、各组元体积分数、比表面积和孔径分布等 电极特征信息。连通性表征的是微结构中各相是 否有良好接触，从而利于物质的传输或传导。不 连通的相(死孔或孤岛)对相应的传输或传导过程 无贡献。连通率定义为连通的孔相(或固体相)节点 数与所有孔相 (或固体相)节点数的比值。采用 Hoshen-Kopelman 聚类算法 ${ }^{[22]}$, 对该石墨负极的 重建微结构(见图 3(c))进行连通性分析, 得到连通 的固相(包括石墨和添加物)和孔相分别占各自总 体积的 $99.60 \%$ 和 $98.00 \%$, 表明该重建结构的连通 性良好。

在重构的石墨负极微结构中, 其各组元体积 分数包括孔隙率、石墨体积分数和添加物体积分 数分别为 $30.5 \%$ 、 $52.0 \%$ 和 $17.5 \%$ ，与初始设定值 基本一致。图 4 是各组元截面平均体积分数沿 $X$ 方向(电极厚度方向)的分布曲线, 各组元体积分 数都有不同程度的局部波动，其中石墨和孔相均 有 0.1 左右的波动幅度，这正好体现了石墨电极 的层状结构特征, 而添加物颗粒较小, 其体积分 数分布相对均匀。

对于孔径的分析, 本研究采用最大内切球法 ${ }^{[23]}$ 。 以欧氏距离转换法 ${ }^{[24-25]}$ 计算内切球直径, 统计分 析可得各尺寸大小孔的相对体积(Relative volume: 各半径大小的孔的体积和总的孔体积的比值)随孔 尺寸大小的分布关系。图 5 示出了如图 3(c) 所示的 重建石墨负极三维微结构内孔径分布, 图中显示 该重建的微结构中最大孔径尺寸 $12 \mu \mathrm{m}$, 整个微结 构内孔径平均尺寸为 $4.45 \mu \mathrm{m}$, 与扁平椭球颗粒的 厚度相当, 反映了负极鳞片状石墨颗粒层叠的结 构特征。
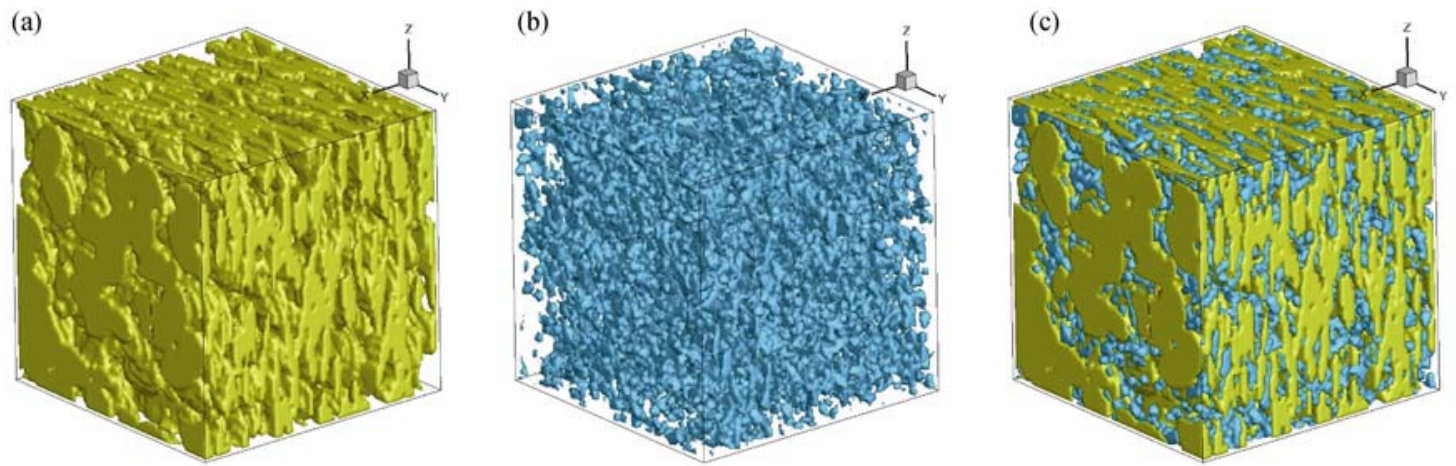

图 3 重建的石墨负极三维微结构

Fig. 3 3D microstructure of reconstructed graphite anode

(a) Spatial distribution of graphite; (b) Spatial distribution of solid additive; (c) Spatial distribution and topological relation of graphite anode microstructure consisting of 3 components. Yellow denotes graphite, blue solid additive and transparent pore or electrolyte 


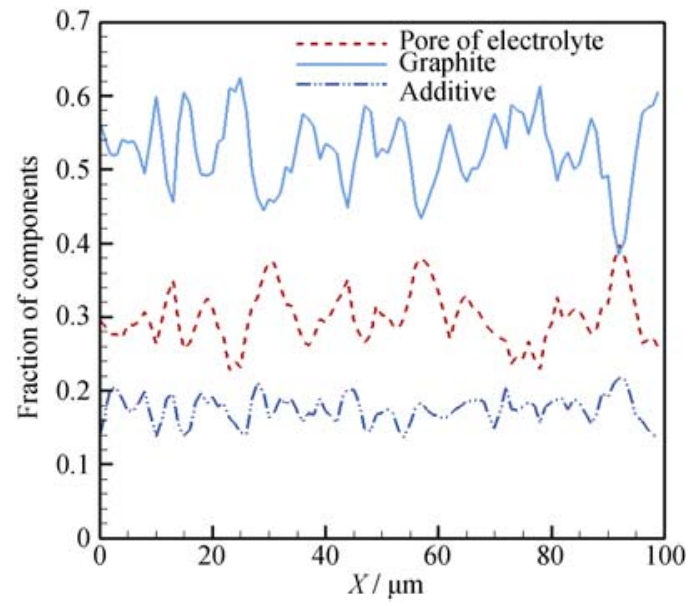

图 4 各相体积分数在沿 $X$ 轴方向(电极厚度方向)的分布 Fig. 4 Distribution of volume fraction of each component along $X$ direction (i.e. the electrode thickness direction)

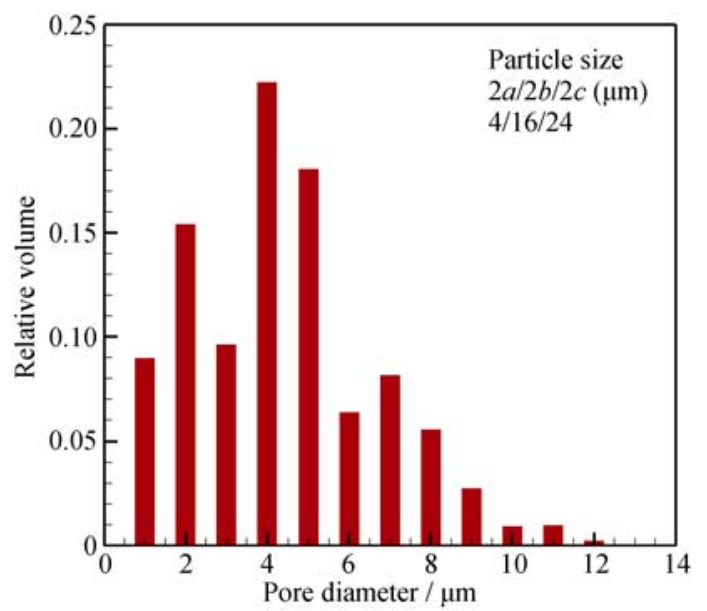

图 5 重建石墨负极内微结构孔径分布

Fig. 5 Pore size distribution inside the reconstructed graphite anode

\section{3 石墨颗粒尺寸对电极微结构特性的影响}

为了探究石墨颗粒尺寸对电极微结构特性影响, 采用基于椭球颗粒的 SA 法, 保持孔隙率、体积分数 和两点相关函数不变, 改变椭球颗粒的尺寸(即改
变 $a 、 b 、 c)$, 重建了多个不同的微结构, 并对重建 微结构进行特征分析, 深入探讨颗粒尺寸对电极特 性的影响, 表 1 汇总了电极微结构平均孔径、比表 面积和连通率随颗粒尺寸的变化。

随着颗粒尺寸的增大, 重建微结构的平均孔径 也不断增大。若两种椭球颗粒的体积相同时，由极 半径更小的椭球颗粒堆积而成的微结构, 其平均孔 径更小(比较颗粒尺寸为 $4 / 8 / 24 \mu \mathrm{m}$ 和 $2 / 16 / 24 \mu \mathrm{m}$ 的 情况)，如表 1 所示。为更加清楚地观察出颗粒尺寸 对重建微结构内孔径分布的影响，图 6 给出了由不 同尺寸的颗粒堆积而成的微结构内的孔径分布图, 从图中可以看出，赤道半径越大，孔隙尺寸分布趋 于向右靠近; 相对于改变极半径, 改变椭球颗粒的 两个赤道半径的长度对孔径分布和平均孔径的影响 相对较小, 这主要是由于椭球颗粒的极半径越小, 颗粒越扁平，颗粒层与层之间重叠更紧密、间距更 小，孔隙尺寸自然就更小。

从表 1 还可以看出，椭球颗粒的尺寸越大，石 墨与孔隙之间的界面越小，即电极的比表面积越小; 若两种椭球颗粒体积相同，由极半径较小的椭球颗 粒重建而成的微结构具有更大的表面积，如由尺寸 为 $2 / 16 / 24 \mu \mathrm{m}$ 的椭球颗粒重建而成的微结构的比表 面积为 $563154 \mathrm{~m}^{-1}$, 而由尺寸为 $4 / 8 / 24 \mu \mathrm{m}$ 的椭球颗 粒重建而成的微结构的比表面积仅为 $479619 \mathrm{~m}^{-1}$ 。 此外，从表 1 还可以看出基于椭球颗粒的 SA 法重建 的负极微结构具有较好的连通性，固相连通率均在 $99.6 \%$ 以上，孔相连通率均超过 $95 \%$ 。由尺寸为 $2 / 16 / 24 \mu \mathrm{m}$ 的椭球颗粒重建而成的微结构的固相连 通率最大, 达到 $99.86 \%$, 但此时由于椭球颗粒的极 半径偏小，即颗粒较为扁平，且交错重叠，从而使 得重建微结构的孔隙空间更易破碎，进而导致孔相 连通率偏小; 由尺寸为 $4 / 8 / 16 \mu \mathrm{m}$ 的椭球颗粒重建 而成的微结构的孔相连通率最大，达到 $98.68 \%$ 。

根据上述分析可知, 石墨颗粒尺寸对电极微结

表 1 颗粒尺寸变化对重建微结构特性的影响

Table 1 Effects of particle size on microstructural characteristics of the reconstructed anode

\begin{tabular}{ccccc}
\hline \multirow{2}{*}{ Particle size $(2 a / 2 b / 2 c) / \mu \mathrm{m}$} & Average pore diameter $/ \mu \mathrm{m}$ & Specific surface area $/ \mathrm{m}^{-1}$ & \multicolumn{2}{c}{ Connectivity $/ \%$} \\
\cline { 3 - 5 } & & & 539430 & 99.62 \\
\hline $4 / 8 / 16$ & 3.88 & 479619 & 99.73 & 98.68 \\
$4 / 8 / 24$ & 4.45 & 456897 & 99.60 & 98.42 \\
$4 / 16 / 24$ & 4.80 & 437198 & 99.04 & 98.00 \\
$4 / 16 / 32$ & 4.87 & 418739 & 99.62 & 97.66 \\
$4 / 24 / 32$ & 3.26 & 563154 & 99.86 & 95.86 \\
\hline
\end{tabular}




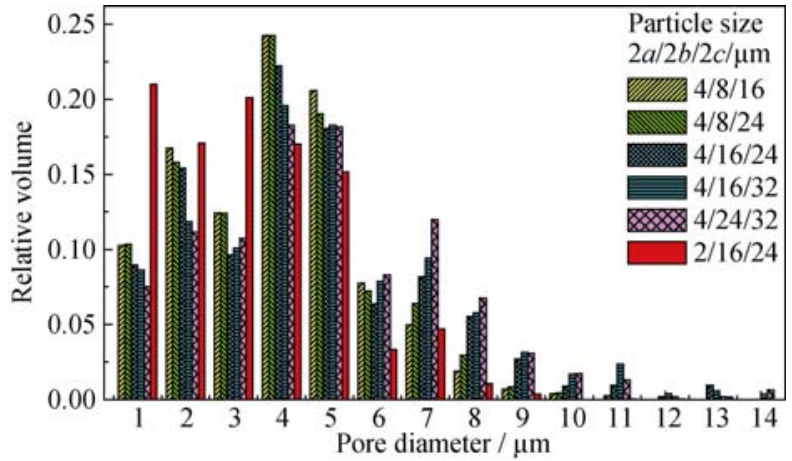

图 6 颗粒尺寸对重构微结构内孔径分布的影响

Fig. 6 Pore size distribution for microstructures reconstructed with particles of different sizes

构都有很大的影响, 重建微结构的平均孔径随颗粒 尺寸的增大而增大，比表面积随尺寸的增大而减小; 相对于赤道半径, 椭球颗粒的极半径对重建电极微 结构特性(连通率、比表面积以及孔径分布等)的影 响更为明显。上述结果可为制备出性能更为优良的 石墨负极提供参考。

\section{4 结论}

1) 基于椭球颗粒的 SA 法考虑了实际石墨负极 的层叠状各向异性和颗粒的鳞片状形态, 重建结构 与实际石墨电极内的微结构形态相符合。

2) 重建过程中, 通过添加约束条件减少了固 体颗粒在孔隙中的孤立悬空, 特别是固体添加物颗 粒, 在重建时要求均匀地紧贴在石墨表面, 因此重 建微结构中各组分相均具有良好的连通性。

3) 石墨颗粒尺寸对重建微结构特性有重要影 响: 椭球颗粒的尺寸越大, 平均孔径越大, 比表面 积越小; 相对于赤道半径, 石墨颗粒的极半径对电 极微结构特性影响更为显著。

下一步, 本课题组将针对重建电极内耦合电化 学反应的多物理传输过程, 建立介观孔或颗粒尺度 数值模型, 预测电极有效传输物性(包括离子/电子 的传导系数、离子/原子扩散系数以及热扩散系数 等), 揭示其内部相关物理-化学机制, 设计优化石 墨负极的介观微结构。

\section{参考文献:}

[1] WEN J W, YU Y, CHEN C H. A review on lithium-ion batteries safety issues: existing problems and possible solutions. Mater. Express, 2012, 2(3): 197-212.

[2] LI Z, HUANG J, LIAW BY, METZLER V, et al. A review of lithium deposition in lithium-ion and lithium metal secondary batteries.
J. Power Sources, 2014, 254: 168-182.

[3] YANG L J, CHENG X Q, GAO Y Z, et al. Lithium compound deposition on mesocarbon microbead anode of lithium ion batteries after long-term cycling. ACS Appl. Mater. Interfaces, 2014, 6(15): 12962-12970.

[4] GUY D, LESTRIEZ B, BOUCHET R, et al. Improvement of lithium battery performance through composite electrode microstructure optimization. Ionics, 2004, 10(5/6): 443-449.

[5] FATTAKHOVA D, KAVAN L, KRTIL P. Lithium insertion into titanium dioxide (anatase) electrodes: microstructure and electrolyte effects. J. Solid State Electrochem., 2001, 5(3): 196-204.

[6] JIANG F M, ZENG J B, WU W. Design and optimization for lithiumion battery electrode: mesoscale numerical model. Advanced Materials Industry, 2011, 12: 2-6.

[7] ZENG J B, JIANG F M. A mesoscale smoothed particle hydrodynamics model for lithium-ion batteries. Acta Phys. -Chim. Sin., 2013, 29(11): 2371-2384.

[8] ZENG J B, WU W, JIANG F M. Smoothed particle hydrodynamics prediction of effective transport coefficients of lithium-ion battery electrodes. Solid State Ionics, 2014, 260(1): 76-85.

[9] QUIBLIER J A. A new three-dimensional modeling technique for studying porous media. J. Colloid Interface Sci., 1984, 98(1): 84-102.

[10] YEONG C L Y, TORQUATO S. Reconstructing random media. Phys. Rev., 1998, 57(1): 495-506.

[11] ČAPEK P, HEJTMÁNEK V, BRABEC L, et al. 3D stochastic replicas of porous solids: a way to improve predicted diffusivity. Diffusion-Fundamentals, 2009, 91: 1-4.

[12] KIM S H, PITSCH H. Reconstruction and effective transport properties of the catalyst layer in PEM fuel cells. J. Electrochem. Soc., 2009, 156(6): 673-681.

[13] WU W, JIANG F M. Simulated annealing reconstruction and characterization of a $\mathrm{LiCoO}_{2}$ lithium-ion battery cathode. Chin. Sci. Bull., 2013, 58(36): 4692-4695.

[14] WU W, JIANG F M. Microstructure reconstruction and characterization of PEMFC electrodes. Int. J. Hydrogen Energy, 2014, 39(28): 15894-15906.

[15] SIDDIQUE N A, LIU F. Process based reconstruction and simulation of a three-dimensional fuel cell catalyst layer. Electrochimica Acta, 2010, 55(19): 5357-5366.

[16] WU W, JIANG F M, CHEN Y, et al. 3D monte carlo reconstruction and characterization of $\mathrm{LiCoO}_{2}$ cathode. Journal of Inorganic Materials, 2013, 28(11): 1243-1247.

[17] STEPHENSON D E, HARTMAN E M, HARB J N, et al. Modeling of particle-particle interactions in porous cathodes for lithium-ion batteries. J. Electrochem. Soc., 2007, 154(12): A1146-A1155.

[18] MARKERVICH E, SALITRA G, LEVI M D, et al. Capacity fad- 
ing of lithiated graphite electrodes studied by a combination of electroanalytical methods, Raman spectroscopy and SEM. J. Power Sources, 2005, 146(1/2): 146-150.

[19] 吴其修, 安秀梅, 刘正荣. 锂离子电池复合碳负极材料及其制 备方法. 中国 C01B31/02, CN 200610060814, 2006.05.19.

[20] 肖 峰, 孙华军. 一种锂离子二次电池的负极及包括该负极的锂 离子二次电池. 中国 H01M10/40, CN 200510002208, 2005. 01. 17 .

[21] PENG P, SUN Y Q, JIANG F M. Thermal analyses of $\mathrm{LiCoO}_{2}$ lithium-ion battery during oven tests. Heat Mass Transfer., 2014, 50(10): 1405-1416

[22] BABALIEVSKI F. Cluster counting: the Hoshen-Kopelman algorithm versus spanning tree approaches. J. Modern Phys. , 1998, 9(1): 43-60.

[23] DELERUE J F, PERRIER E, YU Z Y, et al. New algorithms in 3D image analysis and their application to the measurement of a spatialized pore size distribution in soils. Phys. Chem. Earth A: Solid Earth Geodesy, 1999, 24(7): 639-644.

[24] CHEOLWOONG L, BO Y, YIN L L, et al. Geometric characteristics of three dimensional reconstructed anode electrodes of lithiumion batteries. Energies, 2014, 7(4): 2558-2572.

[25] THIELE S, ZENGERLE R, ZIEGLER C. Nano-morphology of a polymer electrolyte fuel cell catalyst layer-imaging, reconstruction and analysis. Nano Res., 2011, 4(9): 849-860. 